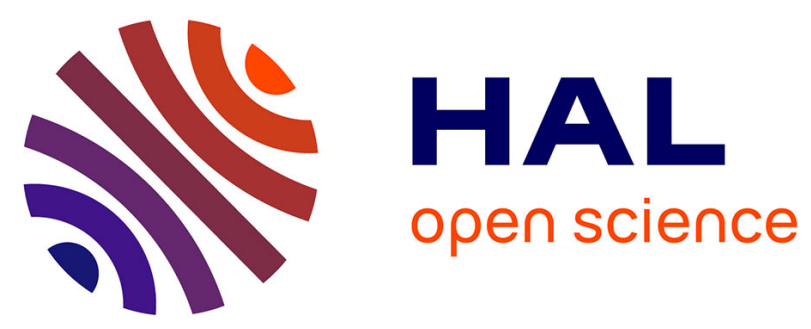

\title{
Variability in fluence and spectrum of high-energy photon bursts produced by lightning leaders
}

Sébastien Celestin, Wei Xu, Victor P. Pasko

\section{To cite this version:}

Sébastien Celestin, Wei Xu, Victor P. Pasko. Variability in fluence and spectrum of high-energy photon bursts produced by lightning leaders. Journal of Geophysical Research Space Physics, 2015, 120 (12), pp.10712-10723. 10.1002/2015JA021410 . insu-01351316

\section{HAL Id: insu-01351316 https://hal-insu.archives-ouvertes.fr/insu-01351316}

Submitted on 1 Dec 2016

HAL is a multi-disciplinary open access archive for the deposit and dissemination of scientific research documents, whether they are published or not. The documents may come from teaching and research institutions in France or abroad, or from public or private research centers.
L'archive ouverte pluridisciplinaire HAL, est destinée au dépôt et à la diffusion de documents scientifiques de niveau recherche, publiés ou non, émanant des établissements d'enseignement et de recherche français ou étrangers, des laboratoires publics ou privés. 


\section{Journal of Geophysical Research: Space Physics}

\section{RESEARCH ARTICLE \\ 10.1002/2015JA021410 \\ Variability in fluence and spectrum of high-energy photon bursts produced by lightning leaders}

Key Points:

- X-ray burst spectrum converges to

TGF spectrum as the potential drop increases

- For strong potential drops, the model leads to fluence and spectrum consistent with TGF observations

- Based on the results, we speculate

that TGFs are a fraction of a much

larger distribution

Correspondence to:

S. Celestin,

sebastien.celestin@cnrs-orleans.fr

\section{Citation:}

Celestin, S., W. Xu, and V. P. Pasko (2015), Variability in fluence and spectrum of high-energy photon bursts produced by lightning leaders, J. Geophys. Res. Space

Physics, 120, 10,712-10,723,

doi:10.1002/2015JA021410.

Received 30 APR 2015 Accepted 9 NOV 2015

Accepted article online 13 NOV 2015 Published online 11 DEC 2015

\author{
Sebastien Celestin ${ }^{1}$, Wei $\mathbf{X u}^{2}$, and Victor P. Pasko ${ }^{2}$ \\ ${ }^{1}$ LPC2E, University of Orleans, CNRS, Orleans, France, ${ }^{2}$ Communications and Space Sciences Laboratory, Department of \\ Electrical Engineering, Pennsylvania State University, University Park, Pennsylvania, USA
}

\begin{abstract}
In this paper, we model the production and acceleration of thermal runaway electrons during negative corona flash stages of stepping lightning leaders and the corresponding terrestrial gamma ray flashes (TGFs) or negative cloud-to-ground (-CG) lightning-produced X-ray bursts in a unified fashion. We show how the source photon spectrum and fluence depend on the potential drop formed in the lightning leader tip region during corona flash and how the X-ray burst spectrum progressively converges toward typical TGF spectrum as the potential drop increases. Additionally, we show that the number of streamers produced in a negative corona flash, the source electron energy distribution function, the corresponding number of photons, and the photon energy distribution and transport through the atmosphere up to low-orbit satellite altitudes exhibit a very strong dependence on this potential drop. This leads to a threshold effect causing X-rays produced by leaders with potentials lower than those producing typical TGFs extremely unlikely to be detected by low-orbit satellites. Moreover, from the number of photons in X-ray bursts produced by -CGs estimated from ground observations, we show that the proportionality between the number of thermal runaway electrons and the square of the potential drop in the leader tip region during negative corona flash proposed earlier leads to typical photon fluences on the order of $1 \mathrm{ph} / \mathrm{cm}^{2}$ at an altitude of $500 \mathrm{~km}$ and a radial distance of $200 \mathrm{~km}$ for intracloud lightning discharges producing $300 \mathrm{MV}$ potential drops, which is consistent with observations of TGF fluences and spectra from satellites.
\end{abstract}

\section{Introduction}

X-ray and gamma ray bursts associated with triggered and natural cloud-to-ground lightning discharges have been observed from the ground [e.g., Moore et al., 2001; Dwyer et al., 2003, 2005]. The energy of individual photons in these events varies from a few tens of keV to a few MeV [e.g., Schaal et al., 2012]. Moreover, terrestrial gamma ray flashes (TGFs) detected by satellites involve individual photon energies of a few tens of MeV [Fishman et al., 1994; Smith et al., 2005; Marisaldi et al., 2010a; Briggs et al., 2010], and there is a growing body of evidence that TGFs are correlated with the initial stages of intracloud lightning discharges that transport negative charge upward (intracloud (+IC)) [e.g., Stanley et al., 2006; Shao et al., 2010; Lu et al., 2011; Connaughton et al., 2010, 2013; Cummer et al., 2011, 2015; Østgaard et al., 2013].

These high-energy phenomena are believed to be caused by the production of runaway electrons. Runaway electrons are energetic electrons with decreased probability of collision as a result of their high energy, so that they gain more energy from a given external electric field than the energy they deposit through collisions [e.g., Dwyer et al., 2012]. As a result, these electrons accelerate on average as long as they propagate under a sufficiently strong electric field. For electrons propagating in neutral air at ground level, electron collisions result in a specific dynamic friction with a maximum of $\sim 260 \mathrm{keV} / \mathrm{cm}$ corresponding to an energy of $\sim 130 \mathrm{eV}$ [e.g., see Moss et al., 2006, Figure 2]. Beyond that energy, the friction decreases with increasing electron energy up to the MeV domain. This maximum in the dynamic friction makes it possible to produce a large number of runaway electrons if the applied field is strong enough. This process is usually named "thermal runaway" and was described by Gurevich [1961].

The dynamic friction in air also presents a minimum for electrons with an energy of $\sim 1.3 \mathrm{MeV}$. In air at ground level, this minimum is approximately $2.1 \mathrm{keV} / \mathrm{cm}$. As a result, electrons with sufficiently high energy propagating in a field greater than the minimum friction are runaway electrons [Wilson, 1925]. In fact, mostly due to elastic scattering, the runaway threshold electric field is $E_{\text {run }} \simeq 2.8 \mathrm{kV} / \mathrm{cm}$ in air at ground level
O2015. American Geophysical Union. All Rights Reserved. 
[e.g., Dwyer et al., 2012]. While most of ionizing collisions produced by runaway electrons result in low-energy secondary electrons that are not runaway electrons, there is a probability that a secondary runaway electron be produced. This situation occurs if the electric field extends over a region that is larger than the corresponding mean-free path of such an ionizing collision. This avalanche mechanism was first reported by Gurevich et al. [1992] and is now termed relativistic runaway electron avalanche (RREA) [see Dwyer et al., 2012, and references therein].

Average TGF spectra are very well reproduced by RREA models involving acceleration of runaway electrons in large-scale (on the order of kilometers) ambient electric fields produced by thunderstorms with amplitudes greater than the abovementioned runaway threshold field $E_{\text {run }} \simeq 2.8 \times N / N_{0} \mathrm{kV} / \mathrm{cm}$, where $N_{0}$ is the ground level air density and $N$ is the air density at a given altitude [Dwyer and Smith, 2005]. It has been theoretically shown that photon spectra produced by RREAs in large-scale homogeneous electric fields exhibit a robust characteristic exponential high-energy cutoff $(\sim 7 \mathrm{MeV})$ in the high-energy part of electron energy distribution function [see Dwyer et al., 2012, and references therein]. This property weakly depends on the production altitude and the magnitude of the large-scale homogeneous electric field that sustains the RREA [e.g., Babich et al., 2004; Dwyer and Smith, 2005]. This signature should therefore be present in the bremsstrahlung photon spectra produced by RREAs and observed from low-orbit satellites [Dwyer and Smith, 2005]. The fluence of TGFs is believed to be too high to be produced by RREAs seeded by natural background radiation or cosmic ray air showers alone, and as a consequence, relativistic feedback mechanisms have been introduced [Dwyer, 2008]. If the ambient field is sufficiently strong, different kinds of large-scale discharges driven by relativistic particles have been predicted to be produced in thunderstorms using numerical models [Liu and Dwyer, 2013].

X-ray bursts have been observed to be produced during the formation of new leader steps in negative cloud-to-ground lightning [Dwyer et al., 2005]. Although the energy of these X-ray bursts was found to be insufficient for the RREA mechanism to be involved [Dwyer et al., 2005], they can be explained by the production and acceleration of thermal runaway electrons in the vicinity of the leader tip during negative corona flash stages of stepping lightning leaders and the subsequent production of bremsstrahlung [Celestin and Pasko, 2011; Xu et al., 2014]. The same phenomenon could be at the origin of the production of TGFs under the assumption that high-potential intracloud leaders are involved [Celestin and Pasko, 2011]. Indeed, a very good agreement between theoretical predictions and the average TGF spectrum measured by the Reuven Ramaty High Energy Solar Spectroscopic Imager (RHESSI) satellite has been obtained for lightning discharges forming potential drops in the leader tip region greater than $100 \mathrm{MV}$ [Xu et al., 2012]. In these high-potential conditions, multiplication of high-energy electrons is present in the electron acceleration region, which therefore leads to similar spectra as those resulting from RREA models involving acceleration of runaway electrons in large-scale ambient electric fields. In fact, for lower potential drops, the photon spectrum depends strongly on the potential drop in the lightning leader tip region, and for potential drops below $100 \mathrm{MV}$, the high-energy part of the average TGF RHESSI spectrum (>10 MeV) is not well reproduced [Xu et al., 2012].

In the following, we distinguish the theory of TGF production by feedback-enhanced RREAs seeded by cosmic ray secondary electrons in large-scale ambient electric fields from that of direct thermal runaway electron production/acceleration and their corresponding avalanches in the transient electric field produced in the lightning leader tip region, as they lead to different testable predictions. For example, in the latter theory, the TGF source is located in a region around the lightning leader tip spanning a few hundreds of meters, whereas feedback-enhanced RREA-based models assume that regions with unusually strong ambient electric fields in thunderstorms extend over distances of several kilometers [e.g., Liu and Dwyer, 2013]. Additionally, optical emissions produced by these two mechanisms are different [Xu et al., 2015]. Another possible discrimination between theoretical models of TGF production might be performed on the basis of TGF spectra analysis. Since the model based on RREAs in large-scale ambient electric fields predicts a robust TGF spectrum observed by satellites and the leader-based model results are strongly dependent on the leader potential (see discussion below), it might be possible to determine which of the production model is valid based on the intrinsic variation of individual TGF spectra measured.

In this paper, we model the production and acceleration of high-energy electrons during negative corona flash stages of stepping intracloud lightning leaders and the corresponding TGFs and CG lightning-related $X$-ray bursts in a unified fashion. We show how the source photon spectrum and fluence depend on the potential drop formed in the lightning leader tip region and how the X-ray burst spectrum progressively converges toward a typical TGF spectrum as the potential drop increases. Additionally, we show that the number 


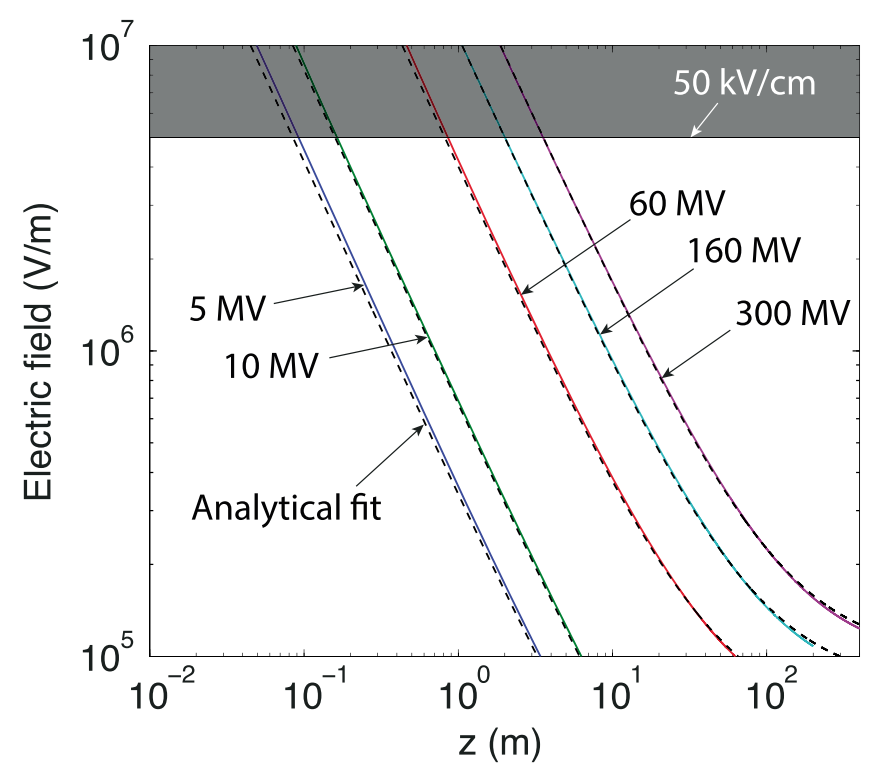

Figure 1. Electric field with respect to the distance from the leader tip calculated using the method of moments. The dashed lines represent analytical fits of the numerical solutions calculated using equation (1). Electric fields with amplitudes greater than $50 \mathrm{kV} / \mathrm{cm}$ are not considered in this study (grey area) as they could only be present on very short timescales. In the Monte Carlo simulations, electrons are injected at the location where the field is equal to $50 \mathrm{kV} / \mathrm{cm}$.

of streamers produced in a negative corona flash, the source electron energy distribution function, the corresponding number of photons, and the photon energy distribution and transport through the atmosphere up to low-orbit satellite altitudes exhibit a very strong dependence on this potential drop. This leads to a threshold effect causing X-rays produced by leaders with potentials lower than those producing typical TGFs extremely unlikely to be detected by low-orbit satellites. Furthermore, we show that the leader-based model allows a continuous scaling in the number of produced high-energy photons from values characteristic for CG lightning-related bursts up to values typical for TGFs that are consistent with the observed fluences and spectra.

\section{Model Formulation}

We first calculate the electric field produced in the vicinity of lightning leader tips during the negative corona flash stage, i.e., when a new leader step has just formed and the streamer zone is not yet fully developed. Hence, at this point, the developing streamers do not efficiently screen out the electric field produced by the leader. The lightning leader is thus considered to be a perfectly conducting cylinder aligned with the ambient large-scale electric field in the thunderstorm. The polarization of the leader in the ambient field results in a specific charge distribution that we calculate using the method of moments [Balanis, 1989, p. 670]. The leader radius is set as $1 \mathrm{~cm}$ in all the cases studied in this paper [e.g., see Rakov and Uman, 2003, p. 134]. The charge distribution on the leader thus depends on two parameters: the length of the leader $I$ and the magnitude of the ambient field $E_{0}$. These two quantities are related to the potential difference between the leader tip and the ambient potential, which is directly connected to the potential drop in the leader tip region (named "potential drop" in the following for the sake of simplicity), through the simple relation $U_{1}=E_{0} I / 2$ [Bazelyan and Raizer, 2000, p. 54]. In the present paper, we consider five cases corresponding to $U_{1}=5 \mathrm{MV}(I=1 \mathrm{~km}$, $\left.E_{0}=0.1 \mathrm{kV} / \mathrm{cm}\right), U_{1}=10 \mathrm{MV}\left(I=2 \mathrm{~km}, E_{0}=0.1 \mathrm{kV} / \mathrm{cm}\right), U_{\text {I }}=60 \mathrm{MV}\left(I=2 \mathrm{~km}, E_{0}=0.6 \mathrm{kV} / \mathrm{cm}\right), U_{\text {I }}=160 \mathrm{MV}$ $\left(I=4 \mathrm{~km}, E_{0}=0.8 \mathrm{kV} / \mathrm{cm}\right)$, and $U_{I}=300 \mathrm{MV}\left(I=6 \mathrm{~km}, E_{0}=1 \mathrm{kV} / \mathrm{cm}\right)$.

From the charge distribution obtained numerically through the method of moments, we directly calculate the electric field produced by the leader additionally to the ambient field and use this result in our Monte Carlo simulations for electrons. For each potential drop studied in this paper, the electric field is shown in Figure 1 with respect to the distance from the leader tip along the axis of symmetry of the system. An analytical fit of 
the $z$ component of the electric field (i.e., parallel component to the leader channel) is found (see Figure 1) using the following expression:

$$
E_{z}(r, z)=\frac{\alpha U_{1}}{\left(r^{2}+z^{2}\right)^{\frac{\beta}{2}}}+E_{0}
$$

where $\alpha=0.066$ in SI units and $\beta \simeq 1.1$. We note that the agreement is particularly good along the axis of symmetry $(r=0)$ in the vicinity of the lightning leader tip. For example, in the case $U_{1}=300 \mathrm{MV}$, the relative error is lower than $3 \%$ along the axis and lower than $6 \%$ in the whole domain. It is interesting to note that this analytical fit offers a good estimate of the electric field for the broad range of ambient fields and potential drops used in this paper. We have not empirically found such a simple representation for the $r$ component of the electric field (i.e., perpendicular component to the leader channel).

To capture the dynamics of the high-energy electrons produced in the vicinity of lightning leaders tip, we use a Monte Carlo model. This model simulates the dynamics of electrons propagating in air with energies from sub-eV to GeV. The model has been used in recent studies to quantify thermal runaway electron emissions from streamer discharges [Celestin and Pasko, 2011], properties of RREAs in homogeneous fields, and the acceleration of electrons in inhomogeneous fields produced by lightning leaders [Celestin et al., 2012; Xu et al., 2012, 2014]. The initial energy of the thermal runaway electrons is defined as $65 \mathrm{keV}$ because those are believed to be produced by expanded streamers developing in the vicinity of the leader tip during the negative corona flash process [Celestin and Pasko, 2011]. However, as electrons strongly accelerate in the field produced by the leader tip, the exact value of the initial electron energy chosen is unimportant as long as the initial electrons are in the runaway regime in a given leader electric field. The initial location of electrons is defined so that the corresponding electric field is $50 \mathrm{kV} / \mathrm{cm}$, which is a reasonable field magnitude at the leader tip [Bazelyan and Raizer, 2000, pp. 67-68]. This upper limit set on the electric field is important because following the static vacuum solution given by the method of moments, extremely strong electric fields are obtained close to the leader tip (see Figure 1). We assume that these strong fields greater than $50 \mathrm{kV} / \mathrm{cm}$ at ground level would not be present if the full dynamics of the stepping process were resolved in our model (see discussion in Celestin and Pasko [2011]). This strong assumption is made in order to simplify the highly complex time dynamics of the leader-streamer zone system during the negative corona flash. Therefore, the full details of the time dynamics of acceleration of the high-energy electrons producing an X-ray burst cannot be reproduced.

Hence, in the $5 \mathrm{MV}, 10 \mathrm{MV}, 60 \mathrm{MV}, 160 \mathrm{MV}$, and $300 \mathrm{MV}$ cases, the initial electron positions are, respectively, set as $8 \mathrm{~cm}, 15 \mathrm{~cm}, 90 \mathrm{~cm}, 2 \mathrm{~m}$, and $3.5 \mathrm{~m}$ from the leader tip. The corresponding electric potential drops between these initial locations and the point beyond which all electrons start decelerating (where the electric field becomes lower than $E_{\text {run }} \simeq 2.8 \mathrm{kV} / \mathrm{cm}$ at ground level) are approximately $1.1 \mathrm{MV}, 2 \mathrm{MV}, 11 \mathrm{MV}, 28 \mathrm{MV}$, and $53 \mathrm{MV}$, respectively. These potential drops are directly related to the maximum energy that electrons can gain. However, in the present paper we have chosen to report our results as functions of the potential difference $U_{1}$ between the potential at the leader tip and the ambient potential at the same position because it is more directly connected to physical properties of the causative lightning discharge.

For the sake of simplicity, Monte Carlo simulations of electrons are performed in ground level air density $N=2.688 \times 10^{25} \mathrm{~m}^{-3}$.

Figure 2 is an illustration of thermal runaway electrons produced by negative streamers during the negative corona flash process subsequent to a leader step formation and their further acceleration in the field produced by the lightning leader. The deflections of these electrons by nuclei of nitrogen and oxygen molecules produce bremsstrahlung radiation. The corresponding instantaneous photon energy spectrum $S\left(\varepsilon_{\gamma}, t\right)$ (in units of $\left.\mathrm{eV}^{-1} \mathrm{~s}^{-1}\right)$ is obtained from the instantaneous normalized energy distribution of electrons $f(\varepsilon, t)$ at any moment of time using the Bethe-Heitler differential cross section $\frac{\mathrm{d} \sigma_{\gamma}}{\mathrm{d} \varepsilon_{\gamma}}\left(\varepsilon, \varepsilon_{\gamma}\right)$ [e.g., Lehtinen, 2000, pp. 45-49], where $\varepsilon$ is the kinetic energy of the electron and $\varepsilon_{\gamma}$ is the energy of the produced photon

$$
S\left(\varepsilon_{\gamma}, t\right)=N \int f(\varepsilon, t) \frac{\mathrm{d} \sigma_{\gamma}}{\mathrm{d} \varepsilon_{\gamma}}\left(\varepsilon, \varepsilon_{\gamma}\right) v_{\mathrm{e}}(\varepsilon) \mathrm{d} \varepsilon
$$

where $v_{\mathrm{e}}(\varepsilon)=c \sqrt{1-\left(1+\frac{\varepsilon}{m c^{2}}\right)^{-2}}$ is the relativistic speed of an electron with energy $\varepsilon$ and rest mass $m$, and $c$ is the speed of light in vacuum. 


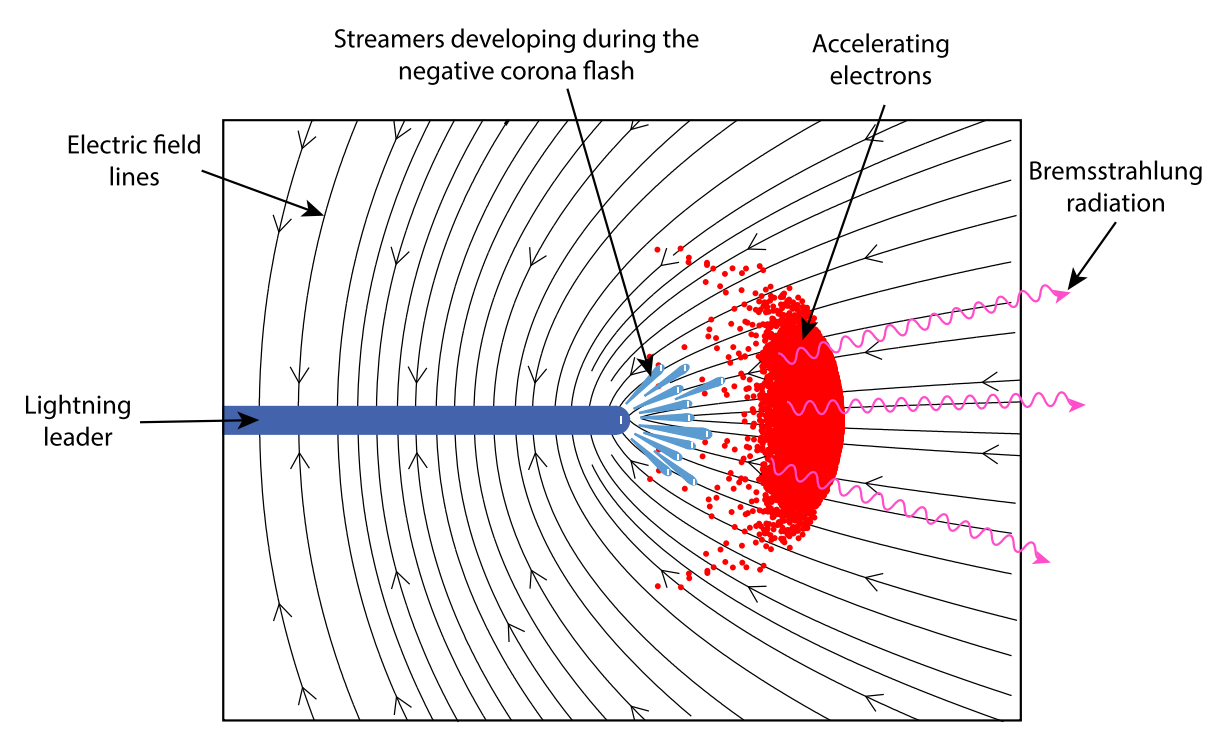

Figure 2. Illustration of the production of runaway electrons and their acceleration in the lightning leader field during the negative corona flash process.

To evaluate the number of photons produced, we calculate the bremsstrahlung photon production frequency per electron $\left\langle v_{\gamma}\right\rangle$ for electrons with energies greater than $\varepsilon_{\min }$ at each moment of time as

$$
\left\langle v_{\gamma}\right\rangle(t)=N \int_{\varepsilon_{\min }}^{+\infty} f(\varepsilon, t) \sigma_{\gamma}(\varepsilon) v_{\mathrm{e}}(\varepsilon) \mathrm{d} \varepsilon
$$

where $\sigma_{\gamma}(\varepsilon)=\int_{\varepsilon_{\min }}^{\varepsilon} \frac{\mathrm{d} \sigma_{\gamma}}{\mathrm{d} \varepsilon_{\gamma}}\left(\varepsilon, \varepsilon_{\gamma}\right) \mathrm{d} \varepsilon_{\gamma}$ is the total cross section for production of bremsstrahlung photons with energies greater than $\varepsilon_{\min }$ by deflections of an electron with energy $\varepsilon>\varepsilon_{\min }$. The bremsstrahlung photon production frequency per electron is then multiplied by the total number of electrons $N_{\mathrm{e}}(t)$ with energies greater than $\varepsilon_{\min }$ at each moment of time and then integrated over time up to the time at which no electron with energy greater than $\varepsilon_{\min }$ is left in the simulation. Thus, we obtain the total number of produced photons with energy greater than $\varepsilon_{\min }$

$$
N_{\gamma}=\int_{0}^{+\infty} N_{\mathrm{e}}(t)\left\langle v_{\gamma}\right\rangle(t) \mathrm{d} t
$$

The time-integrated photon spectrum $S\left(\varepsilon_{\gamma}\right)$ is obtained by substituting the instantaneous electron distribution $f(\varepsilon, t)$ with the time-integrated electron distribution $f(\varepsilon)$ (weighting $f(\varepsilon, t)$ by the number of electrons at any moment of time) in equation (2).

In addition, we use a Monte Carlo model to simulate photon transport through the atmosphere in a similar way as that described by Østgaard et al. [2008]. Three main collision types for photons with energies between $10 \mathrm{keV}$ and $100 \mathrm{MeV}$ are considered: photoelectric absorption (main process for energies up to $\sim 30 \mathrm{keV}$ ), Compton scattering (main process from $\sim 30 \mathrm{keV}$ to $\sim 30 \mathrm{MeV}$ ), and electron-positron pair production (main process $>30 \mathrm{MeV}$ ). This model has been validated through calculations of TGF energy spectra based on RREA theory [Xu et al., 2012]. A very good agreement has been obtained with previously published results [e.g., Dwyer and Smith, 2005].

\section{Results}

Figures $3 a$ and $3 b$, respectively, show simulation results of time-integrated electron energy distribution functions (EEDF) for each lightning leader potential drop magnitude and the corresponding time-integrated bremsstrahlung photon spectra at the source (photon transport through the atmosphere is not taken into account at this point) calculated using the formulas described in the previous section. The distributions and spectra shown in Figure 3 are normalized so that integration over the presented energy range yields one. It is clear that, as the potential drop in the leader tip region increases, the electron and photon distributions 

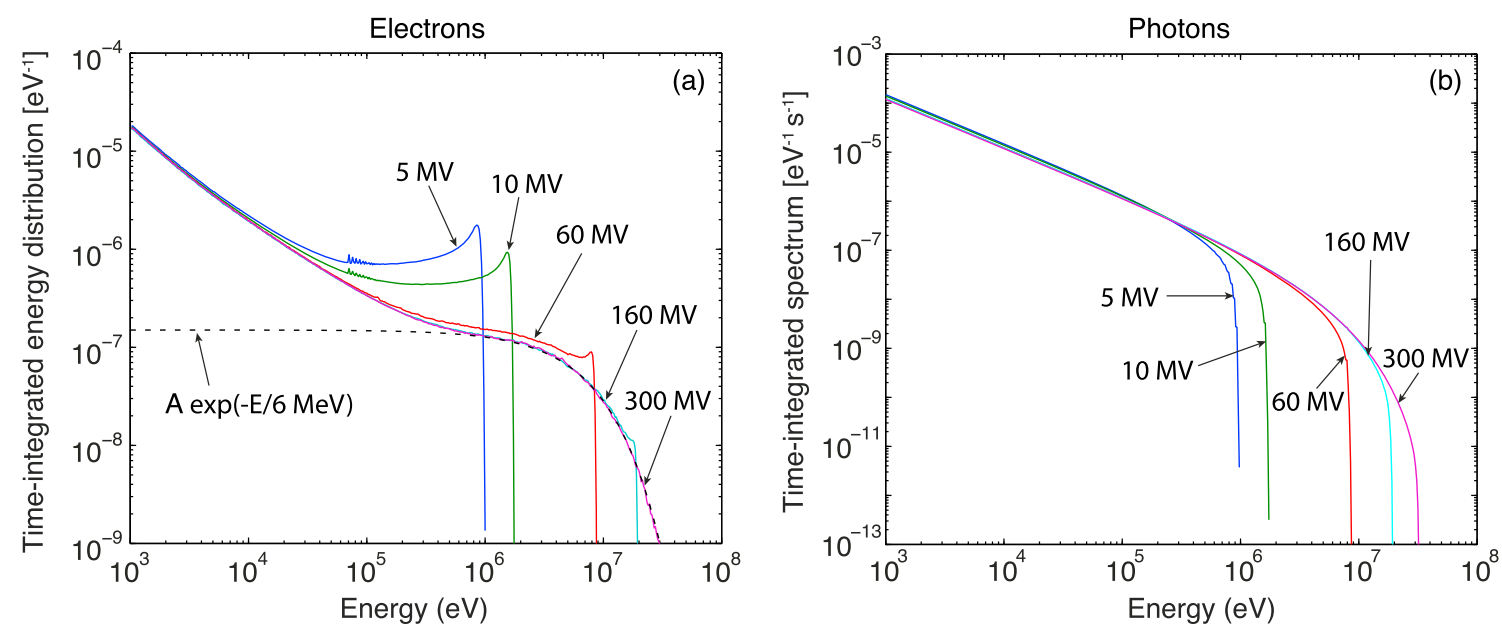

Figure 3. (a) Time-integrated electron energy distribution functions for each lightning leader potential drop magnitude used in the present study. (b) Corresponding bremsstrahlung photon spectra calculated using equation (2).

become more energetic. Although the photon spectra corresponding to different potential drops present similar features (Figure 3b) with significantly different energies, it is interesting to note that the shapes of the electron distributions vary significantly as the potential drop increases. Close to the maximum energy gained by electrons in each case, we can clearly see a peak in the EEDFs corresponding to potential drops for a few tens of megavolts. However, this feature attenuates as the potential drop increases, as it becomes hardly noticeable for the $160 \mathrm{MV}$ case and it is not visible in the $300 \mathrm{MV}$ case.

Figure 4 represents the number of electrons with energies greater than $1 \mathrm{MeV}$ obtained in the simulations per runaway electron initially injected in the system as a function of time. In the $5 \mathrm{MV}$ case, the energy gained by the accelerating electrons does not reach $1 \mathrm{MeV}$. For the $10 \mathrm{MV}$ case, nearly only the thermal runaway electrons that were initially injected gain energies greater than $1 \mathrm{MeV}$, and hence, the number of electrons with energies greater than $1 \mathrm{MeV}$ per electron initially injected is very close to unity. A more significant amplification of high-energy electrons is seen for higher potential drops (see section 4). We emphasize that the results shown in Figure 4 correspond to an instantaneous injection of all the initial thermal runaway electrons in the vicinity of the leader tip. The results shown in Figure 4 therefore represent impulse responses of the system. They can be convolved with the actual time distribution of the production of thermal runaway electrons by streamer discharges in the negative corona flash in order to obtain the true duration of the process. However, the highly complex time dynamics of the leader-streamer zone system during the negative corona flash is not

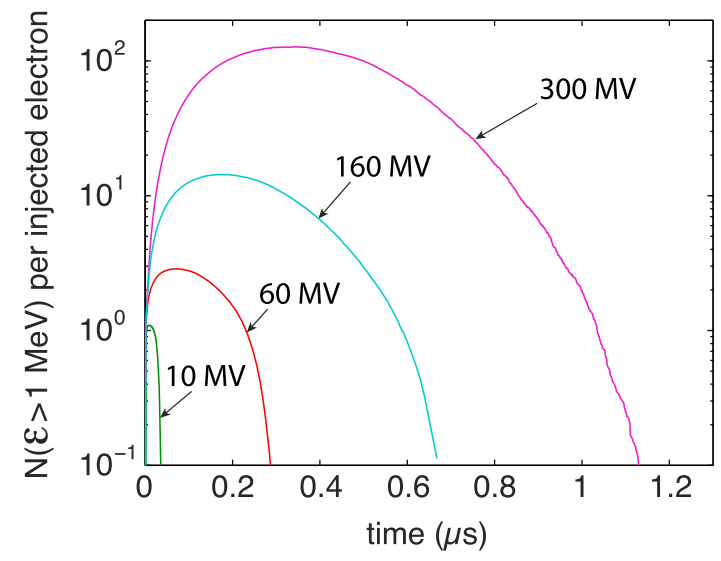

Figure 4. Number of electrons with energies greater than $1 \mathrm{MeV}$ per electron initially injected in the system as a function of time. The true total duration of the process is longer than shown in this figure as these results correspond to impulse responses. 
Table 1. Number of High-Energy Photons

\begin{tabular}{lccc} 
Potential Drop & $\begin{array}{c}>10 \mathrm{keV} \\
\text { (per Injected Electron) }\end{array}$ & $\begin{array}{c}>1 \mathrm{MeV} \\
\text { (per Injected Electron) }\end{array}$ & $\begin{array}{c}\text { Total Number of Photons } \\
{ }^{\mathrm{a}}\end{array}$ \\
\hline $5 \mathrm{MV}$ & 0.086 & 0 & $10^{11}$ \\
$10 \mathrm{MV}$ & 0.21 & 0.0059 & $10^{12}$ \\
$60 \mathrm{MV}$ & 3.3 & 0.53 & $6 \times 10^{14}$ \\
$160 \mathrm{MV}$ & 31.8 & 6.1 & $4 \times 10^{16}$ \\
$300 \mathrm{MV}$ & 404 & 79 & $2 \times 10^{18}$ \\
\hline
\end{tabular}

${ }^{\mathrm{a}} \mathrm{A}$ reference of $10^{11}$ photons with energy $>10 \mathrm{keV}$ in a $5 \mathrm{MV}$ leader case, which corresponds to estimates based on the observations of negative cloud-to-ground discharges. From this reference, we derive the numbers of photons for the other potential drops using the simulation results (see section 4). If it is assumed that $10^{12}$ photons with energies greater than $10 \mathrm{keV}$ are produced instead of $10^{11}$, the total number of photons simply needs to be multiplied by 10 .

resolved in our study, and only a simple limit of $50 \mathrm{kV} / \mathrm{cm}$ is set on the electric field produced at the leader tip (see section 2). Investigation of related scenarios is beyond the scope of the present study.

Table 1 shows the number of photons with energies greater than either $10 \mathrm{keV}$ or $1 \mathrm{MeV}$ per electron initially injected obtained by applying equation (4) to the simulation results presented in this paper and an estimate of the total number of photons produced for different potential drops in the vicinity of the leader tip. The total number of photons is obtained by assuming that $10^{11}$ photons with energies greater than $10 \mathrm{keV}$ are produced in the case of a potential drop of $5 \mathrm{MV}$ (see section 4). The number of thermal runaway electrons $N_{\text {th }}$ initially produced by streamers during the negative corona flash is expected to be proportional to the number of streamers in the streamer zone $N_{s}$, which is theoretically proportional to the square of the potential drop $U_{1}$ in the leader tip region [Celestin and Pasko, 2011]:

$$
N_{\mathrm{th}}=\xi N_{\mathrm{s}}=\xi \frac{\pi \varepsilon_{0} U_{1}^{2}}{2 E_{\mathrm{s}}^{-} q_{\mathrm{s}}}
$$

where $\xi$ is the proportionality constant, $\varepsilon_{0}$ is the permittivity of free space, $q_{\mathrm{s}} \simeq 1 \mathrm{nC}$ is the typical charge in the streamer head [Bazelyan and Raizer, 2000, pp.69-71], and $E_{\mathrm{s}}^{-}$is the critical field for stable propagation of negative streamer. Qin and Pasko [2014] have shown that $E_{s}^{-}$depends on the specific initial conditions leading to streamer formation and generally can be greater than $10 \mathrm{kV} / \mathrm{cm}$, while $\sim 10 \mathrm{kV} / \mathrm{cm}$ represents a good order of magnitude estimate of this field. The proportionality coefficient in equation (5) is obtained from the assumption that $10^{11}$ photons with energies greater than $10 \mathrm{keV}$ are produced in the case of a potential drop of $5 \mathrm{MV}$ (see section 4). Following this procedure, we find that $\xi \simeq 3.4 \times 10^{6}$, which is consistent with the upper limit of $10^{10}$ found by considering that all the streamers eventually constituting the streamer zone emit runaway electrons when they reached their limit of growth [Celestin and Pasko, 2011; Xu et al., 2014]. The total number of photons corresponding to other potential drops is obtained through the assumption that this coefficient does not depend on the potential drop.

Table 2 shows the TGF fluence obtained at an altitude of $500 \mathrm{~km}$ and a radial distance from the source of $200 \mathrm{~km}$ (subsatellite point-to-source distance) as a function of potential drops formed by lightning leaders. To calculate these TGF fluences, we have used our Monte Carlo model simulating the transport of photons

$\begin{aligned} & \text { Table 2. TGF Fluence at an Altitude of } 500 \mathrm{~km} \text { and a Radial } \\
& \text { Distance of } 200 \mathrm{~km} \text { From the Source }\end{aligned}$
\begin{tabular}{lc} 
Potential Drop & Fluence \\
\hline $5 \mathrm{MV}$ & $9 \times 10^{-11} \mathrm{ph} / \mathrm{cm}^{2}$ \\
$10 \mathrm{MV}$ & $5 \times 10^{-9} \mathrm{ph} / \mathrm{cm}^{2}$ \\
$60 \mathrm{MV}$ & $1.5 \times 10^{-4} \mathrm{ph} / \mathrm{cm}^{2}$ \\
$160 \mathrm{MV}$ & $0.02 \mathrm{ph} / \mathrm{cm}^{2}$ \\
$300 \mathrm{MV}$ & $1 \mathrm{ph} / \mathrm{cm}^{2}$ \\
\hline
\end{tabular}

a The TGF source altitude is chosen at $12 \mathrm{~km}$. 


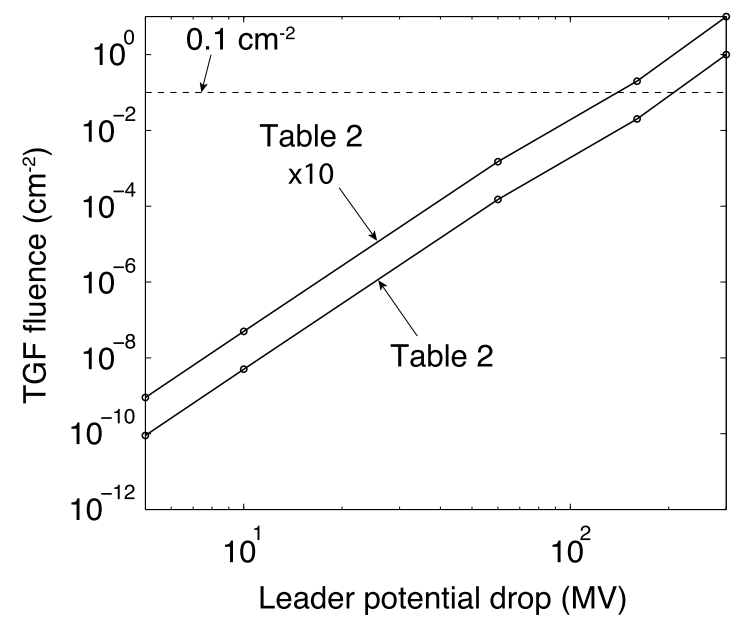

Figure 5. Calculated TGF fluence as a function of the potential drop formed by the causative lightning leader at an altitude of $500 \mathrm{~km}$ and a radial distance of $200 \mathrm{~km}$ from the source. The lower (upper) curve is calculated assuming that $10^{11}\left(10^{12}\right)$ photons with energies greater than $10 \mathrm{keV}$ are produced by a leader forming a potential drop of $5 \mathrm{MV}$ (see Table 2). The dashed line represents an effective current threshold of $0.1 \mathrm{~cm}^{-2}$ for TGF satellite detection.

through the atmosphere and the total number of photons $>10 \mathrm{keV}$ at the source presented in Table 1. The radial distance of $200 \mathrm{~km}$ is chosen because it corresponds approximately to the maximum probability of receiving a TGF photon when considering the variation of photon density with radial distance from the source and the probability for a low-orbit satellite to be at a given radial distance from a TGF source [Celestin and Pasko, 2012]. Additionally, this radial distance corresponds well to typical radial distances of TGF observations by Fermi [e.g., Briggs et al., 2013, Figure 8], AGILE [Marisaldi et al., 2010b, 2014], and RHESSI [e.g., Nisi et al., 2014, Figure 5]. Figure 5 shows the data presented in Table 2 assuming that $10^{11}$ to $10^{12}$ photons with energies greater than $10 \mathrm{keV}$ are produced by a leader forming a potential drop of $5 \mathrm{MV}$.

\section{Discussion}

It is important to note that, in the present work, the leader channel geometry and amplitudes of ambient electric field are assumed in order to lead to given leader potential drop $U_{1}$. At TGF production altitudes, the air density is reduced and so is the ambient electric field. From equation (1), it can be shown that the energy gained by the electrons from their initiation location (corresponding to $E=50 \mathrm{kV} / \mathrm{cm} \times N / N_{0}$ ) to the point where they start decelerating is weakly dependent on the altitude if the ambient electric field amplitude $E_{0}$ scales with the air density as $N / N_{0}$ and if $\beta$ is close to 1 . The acceleration length scales as $\left(N_{0} / N\right)^{1 / \beta}$. Therefore, the energy loss of minimum ionizing electrons over the acceleration region also weakly depends on the source altitude. Assuming that the electric field in the deceleration region is negligible compared to the dynamic friction, the deceleration length scales as $N_{0} / N$. As $\beta \simeq 1$, one sees that the dynamics of electrons remains very similar at different altitudes for a given leader potential drop $U_{1}$. However, the propagation length of the electrons can be significantly increased. For example, a factor of $\sim 4$ should be considered in the propagation length of electrons at $12 \mathrm{~km}$. Since the propagation length approximately scales as $N_{0} / N$, the number of collisions and the number of electrons and photons produced are weakly dependent of the source altitude.

The ambient electric field $E_{0}$ should decrease with the altitude to prevent it from reaching unphysical values. Therefore, using the leader geometry considered in this paper for the sake of simplicity (straight unbranched leader) to obtain high potential drops $U_{1}$ leads to required leader lengths that might be too long. However, we emphasize that the most important lightning parameter assumed here is the potential drop $U_{1}$, and other leader geometries can lead to enhanced potential drops. Mallios et al. [2013] have recently shown that downward positive leaders propagating before the start of the propagation of negative leaders in a bidirectional intracloud discharge could lead to extreme potentials in the lightning leader as compared to the ambient potential at the negative leader tip. This idea is supported by the fact that the threshold for initiation and propagation of positive leaders may be lower than that of negative leaders (see discussion in Mallios et al. [2013, section 4.2]). More specifically, in the case studied by Mallios et al. [2013], a large-scale ambient electric field of $0.7 \mathrm{kV} / \mathrm{cm}$ involved a $300 \mathrm{MV}$ potential drop in front of the leader tip. Those values are consistent with 
those used in the present study. We speculate that such positive and negative lightning leader sequence and lightning geometry would produce extreme electric potential drops and the related TGFs.

When accelerating in the inhomogeneous electric field produced by a lightning leader during negative corona flash, the instantaneous EEDF tends to be very hard in energy due to the nonequilibrium acceleration process as described in Celestin et al. [2012]. After some time, electrons slow down as they reach locations where the electric field produced by the leader becomes weaker and the corresponding instantaneous EEDF becomes softer. This whole dynamics results in the time-integrated distributions shown in Figure 3a. For very high potential drops, the difference between results presented here and results obtained in [Celestin et al., 2012] is that the acceleration process is not stopped after a few tens of nanoseconds to match the AGILE high-energy spectrum [Tavani et al., 2011], but is able to continue up until no electron with energy greater than 1 keV is present in the simulation.

As shown in Figure 3a, a time-integrated EEDF corresponding to strong potential drops resembles the standard RREA distribution with an exponential cutoff. However, as shown in Figure 3a, the exponential cutoff is found at $6 \mathrm{MeV}$, which is lower than the cutoff of $\sim 7 \mathrm{MeV}$ usually presented in the literature [e.g., Dwyer et al., 2012]. We have verified that this is caused by the change of the EEDF during the deceleration phase of the electrons that contributes to the time-integrated distribution. This phase occurs when the electrons reach locations where the electric field produced by the leader becomes weaker than the relativistic runaway threshold field, and as a consequence, the mean electron energy decreases. Although we have verified that this result is consistent with RHESSI TGF average spectrum (see discussion below), this feature may be of interest for further investigation of TGF production mechanisms using future observations. Moreover, from the initiation to the last electron with energy greater than $1 \mathrm{keV}$ present in the simulation, the propagation zone of runaway electrons for ground level air density spans approximately $350 \mathrm{~m}$ for a potential drop of $300 \mathrm{MV}$.

The total number of high-energy photons produced during a negative corona flash event is difficult to obtain from purely theoretical considerations. As of yet, only upper limits had been used [Celestin and Pasko, 2011; Xu et al., 2012, 2014] employing an assumption that every single streamer in a corona flash produces thermal runaway electrons with the maximum possible flux [Celestin and Pasko, 2011]. However, observations indicate that the number of photons with energies greater than $10 \mathrm{keV}$ produced by a negative corona flash of natural -CG lightning leader is on the order of $10^{11}-10^{12}$ [Saleh et al., 2009; Dwyer et al., 2010; Schaal et al., 2012]. Using numerical modeling, Xu et al. [2014] have shown that 5 MV lightning-stepped leaders would produce X-ray spectra similar to observational results of Schaal et al. [2012] in terms of general shape and spectral hardness, and this potential drop is also consistent with the size and electric charge of streamer zones inferred from X-ray observations obtained by Schaal et al. [2014]. It is therefore reasonable to deduce that negative corona flash produced by natural stepped lightning leader with potential drop of $\sim 5 \mathrm{MV}$ would produce $10^{11}-10^{12}$ photons with energies greater than $10 \mathrm{keV}$. This link between modeling and observational results is an important point because it yields the missing proportionality constant $\xi$ between the square of the leader potential drop magnitude and the number of initial thermal runaway electrons (see equation (5)). Once this proportionality constant is known, assuming that it does not depend on the potential drop, one can estimate the number of high-energy photons for any potential drops. The total number of photons produced for different potential drops is presented in Table 1 assuming that $10^{11}$ photons with energies greater than 10 keV are produced in the case of a potential drop of $5 \mathrm{MV}$. If $10^{12}$ photons with energies greater than $10 \mathrm{keV}$ are produced instead of $10^{11}$, the total number of photons in Table 1 simply needs to be multiplied by 10 .

From the results shown in Table 1, we see that a $300 \mathrm{MV}$ potential drop leads to the production of $10^{18}$ photons with energies greater than $10 \mathrm{keV}$. These photons are produced by high-energy electrons propagating over a total distance of $\sim 350 \mathrm{~m}$ from the initiation to extinction. In fact, the electrons accelerate over $\sim 70 \mathrm{~m}$, which corresponds to the location where the electric field magnitude drops below the RREA threshold of $2.8 \mathrm{kV} / \mathrm{cm}$. The number of electrons with energy greater than $1 \mathrm{MeV}$ continues to increase up until they reach $\sim 100 \mathrm{~m}$. These lengths are obtained under ground level air density conditions and would increase for higher altitudes. Given the photon spectrum obtained for this potential drop (Figure $3 b$ ) at the source, using our Monte Carlo code to simulate the photon transport through the atmosphere, we find that, for a source at $12 \mathrm{~km}$ altitude, a satellite with an altitude of $500 \mathrm{~km}$ and a radial distance of $200 \mathrm{~km}$ would measure a photon fluence of $\sim 1 \mathrm{ph} / \mathrm{cm}^{2}$. This estimate assumes an ideal detector and is in agreement with satellite observations of TGFs [e.g., Briggs et al., 2010; Gjesteland et al., 2012]. Additionally, applying the 
RHESSI detector response matrix [Dwyer and Smith, 2005; Grefenstette et al., 2009] (available at http://scipp. ucsc.edu/ dsmith/tgflib_public/data/), we find that the measured satellite photon spectrum at an altitude of $500 \mathrm{~km}$ accumulated for radial distances between 0 and $500 \mathrm{~km}$ is in agreement with the average RHESSI TGF spectrum for TGF sources located between 12 and $15 \mathrm{~km}$ altitude (with a better agreement closer to $15 \mathrm{~km}$ ). This is consistent with results reported by Xu et al. [2012].

As seen in equation (5), the number of thermal runaway electrons produced during a corona flash is theoretically proportional to the square of the potential drop formed in front of the lightning leader tip. The electron energy strongly depends on the leader potential drop (see Figure 3a). The multiplication of runaway electrons and the duration of their propagation in the leader field strongly increase with the leader potential drop (Figure 4). Hence, the number (see Table 1) and energy (see Figure 3b) of bremsstrahlung photons strongly increase with the leader potential drop. Finally, the number of photons that can escape into space depends on the energy and number of photons at the source and is in turn very sensitive to the potential drop in front of the lightning leader as seen in Table 2. We see in this table that if $300 \mathrm{MV} I \mathrm{C}$ lightning leaders are to produce TGFs with a fluence of $1 \mathrm{ph} / \mathrm{cm}^{2}$, a TGF produced by a leader forming a potential drop only a factor of two lower would be invisible to usual detectors in space. We can also see that X-ray bursts produced by ICs with potential drops similar to those of -CGs (between 5 and $10 \mathrm{MV}$ ) would not be detectable from space. From these results, we therefore conclude that the leader producing TGF model predicts that TGFs detected by satellites represent only a small fraction of a much larger distribution, in agreement with existing observation-based analyses [Østgaard et al., 2012]. This is clearly shown in Figure 5, where the dashed line corresponds to a fluence $0.1 \mathrm{ph} / \mathrm{cm}^{2}$, an effective current threshold for TGF detection by satellite. This emphasizes the need of aircraft campaigns to perform measurements as close as possible to the lightning X-ray burst sources [e.g., see Smith et al., 2011; Hansen et al., 2013].

\section{Conclusions}

We summarize the main conclusions of this paper as follows.

1. We have modeled the propagation and multiplication of runaway electrons in the inhomogeneous electric field formed in front of negative lightning leaders during negative corona flash stages. The lightning-formed potential drops studied in this paper vary from $5 \mathrm{MV}$, that are considered to be typical for -CGs, up to $300 \mathrm{MV}$, that would be produced by ICs and be the source of TGFs.

2. We have specifically investigated the dependencies on the leader potential drop of the electron energy distribution, the electron multiplication and propagation, the photon spectrum at the source, and the photon fluence as observed by satellite.

3. It is found that the source photon spectra become more energetic with increasing lightning leader potential drops in a continuous fashion and are similar to photon spectra associated with RREAs in large-scale ambient electric fields for potential drops greater than $\sim 200 \mathrm{MV}$, except for a slightly lower exponential cutoff due to the slowing down of electrons outside the high-field region in the vicinity of the leader tip, in agreement with the average RHESSI TGF spectrum for source altitudes between 12 and $15 \mathrm{~km}$.

4. Using the number of photons in X-ray bursts produced by -CGs estimated from observations as a reference point, we have shown that positive intracloud lightning discharges producing $300 \mathrm{MV}$ potential drops would lead to photon bursts with fluences and spectra consistent with TGF observations. This establishes a clear physical connection between X-ray bursts produced by lightning leaders and TGFs.

Acknowledgments

Sebastien Celestin's research was supported by the French space agency (CNES) as part of the TARANIS mission. This research was also supported by the NSF grant AGS-1106779 to Penn State University. Part of the simulation results presented in this paper has been obtained using the computer cluster at the Centre de Calcul Scientifique en région Centre (CCSC). All data used in this paper are directly available after a request is made to authors S.C. (sebastien.celestin@cnrs-orleans.fr), W.X. (wxx5015@psu.edu), or V.P.P (vpasko@psu.edu).
5. Finally, we have estimated that if lightning leaders producing potential drops close to $300 \mathrm{MV}$ during negative corona flashes are indeed responsible for TGFs, the very strong dependence of the TGF fluence on the potential drop would make TGFs produced by leaders with slightly lower potential drops (e.g., $160 \mathrm{MV}$ ) invisible to usual detectors in space. We therefore conclude that the leader producing TGFs model predicts that TGFs detected by satellites represent only a small fraction of a much larger distribution in agreement with conclusions of observation-based studies already published in the literature.

\section{References}

Babich, L. P., E. N. Donskoy, R. I. II'Kaev, I. M. Kutsyk, and R. A. Roussel-Dupre (2004), Fundamental parameters of a relativistic runaway electron avalanche in air, Plasma Phys. Rep., 30, 616-624.

Balanis, C. A. (1989), Advanced Engineering Electromagnetics, John Wiley, New York.

Bazelyan, E. M., and Y. P. Raizer (2000), Lightning Physics and Lightning Protection, IOP Publishing Ltd, Bristol, U. K., and Philadelphia, Pa. Briggs, M. S., et al. (2010), First results on terrestrial gamma ray flashes from the Fermi Gamma-ray Burst Monitor, J. Geophys. Res., 115, A07323, doi:10.1029/2009JA015242. 
Briggs, M. S., et al. (2013), Terrestrial gamma-ray flashes in the Fermi era: Improved observations and analysis methods, J. Geophys. Res. Space Physics, 118, 3805-3830, doi:10.1002/jgra.50205.

Celestin, S., and V. P. Pasko (2011), Energy and fluxes of thermal runaway electrons produced by exponential growth of streamers during the stepping of lightning leaders and in transient luminous events, J. Geophys. Res., 116, A03315, doi:10.1029/2010JA016260.

Celestin, S., and V. P. Pasko (2012), Compton scattering effects on the duration of terrestrial gamma-ray flashes, Geophys. Res. Lett., 39 , L02802, doi:10.1029/2011GL050342.

Celestin, S., W. Xu, and V. P. Pasko (2012), Terrestrial gamma ray flashes with energies up to $100 \mathrm{MeV}$ produced by nonequilibrium acceleration of electrons in lightning, J. Geophys. Res., 117, A05315, doi:10.1029/2012JA017535.

Connaughton, V., et al. (2010), Associations between Fermi Gamma-ray Burst Monitor terrestrial gamma ray flashes and sferics from the World Wide Lightning Location Network, J. Geophys. Res., 115, A12307, doi:10.1029/2010JA015681.

Connaughton, V., et al. (2013), Radio signals from electron beams in terrestrial gamma ray flashes, J. Geophys. Res. Space Physics, 118, 2313-2320, doi:10.1029/2012JA018288.

Cummer, S. A., G. Lu, M. S. Briggs, V. Connaughton, S. Xiong, G. J. Fishman, and J. R. Dwyer (2011), The lightning-TGF relationship on microsecond timescales, Geophys. Res. Lett., 381, L14810, doi:10.1029/2011GL048099.

Cummer, S. A., F. Lyu, M. S. Briggs, G. Fitzpatrick, O. J. Roberts, and J. R. Dwyer (2015), Lightning leader altitude progression in terrestrial gamma-ray flashes, Geophys. Res. Lett., 42, 7792-7798, doi:10.1002/2015GL065228.

Dwyer, J. R. (2008), Source mechanisms of terrestrial gamma-ray flashes, J. Geophys. Res., 113, D10103, doi:10.1029/2007JD009248.

Dwyer, J. R., and D. M. Smith (2005), A comparison between Monte Carlo simulations of runaway breakdown and terrestrial gamma-ray flash observations, Geophys. Res. Lett., 32, L22804, doi:10.1029/2005GL023848.

Dwyer, J. R., et al. (2003), Energetic radiation during rocket-triggered lightning, Science, 299, 694, doi:10.1126/science.1078940.

Dwyer, J. R., et al. (2005), X-ray bursts associated with leader steps in cloud-to-ground lightning, Geophys. Res. Lett., 32, L01803, doi:10.1029/2004GL021782.

Dwyer, J. R., D. M. Smith, M. A. Uman, Z. Saleh, B. Grefenstette, B. Hazelton, and H. K. Rassoul (2010), Estimation of the fluence of high-energy electron bursts produced by thunderclouds and the resulting radiation doses received in aircraft, J. Geophys. Res., 115, D09206, doi:10.1029/2009JD012039.

Dwyer, J. R., D. M. Smith, and S. A. Cummer (2012), High-energy atmospheric physics: Terrestrial gamma-ray flashes and related phenomena, Space Sci. Rev., 173, 133-196.

Fishman, G., et al. (1994), Discovery of intense gamma-ray flashes of atmospheric origin, Science, 264, 1313-1316.

Gjesteland, T., N. Østgaard, A. B. Collier, B. E. Carlson, C. Eyles, and D. M. Smith (2012), A new method reveals more TGFs in the RHESSI data, Geophys. Res. Lett., 39, L05102, doi:10.1029/2012GL050899.

Grefenstette, B. W., D. M. Smith, B. J. Hazelton, and L. I. Lopez (2009), First RHESSI terrestrial gamma ray flash catalog, J. Geophys. Res., 114, A02314, doi:10.1029/2008JA013721.

Gurevich, A. V. (1961), On the theory of runaway electrons, Sov. Phys. J. Exper. Theor. Phys., 12, 904-912.

Gurevich, A. V., G. M. Milikh, and R. A. Roussel-Dupré (1992), Runaway electron mechanism of air breakdown and preconditioning during a thunderstorm, Phys. Lett. A, 165(5-6), 463-468.

Hansen, R. S., N. Østgaard, T. Gjesteland, and B. Carlson (2013), How simulated fluence of photons from terrestrial gamma ray flashes at aircraft and balloon altitudes depends on initial parameters, J. Geophys. Res. Space Physics, 118, 2333-2339, doi:10.1002/jgra.50143.

Lehtinen, N. G. (2000), Relativistic runaway electrons above thunderstorms, PhD thesis, Stanford Univ., Stanford, Calif.

Liu, N., and J. R. Dwyer (2013), Modeling terrestrial gamma ray flashes produced by relativistic feedback discharges, J. Geophys. Res. Space Physics, 118, 2359-2376, doi:10.1002/jgra.50232.

Lu, G., S. A. Cummer, J. Li, F. Han, D. M. Smith, and B. W. Grefenstette (2011), Characteristics of broadband lightning emissions associated with terrestrial gamma ray flashes, J. Geophys. Res., 116, A03316, doi:10.1029/2010JA016141.

Mallios, S. A., S. Celestin, and V. P. Pasko (2013), Production of very high potential differences by intracloud lightning discharges in connection with terrestrial gamma ray flashes, J. Geophys. Res. Space Physics, 118, 912-918, doi:10.1002/jgra.50109.

Marisaldi, M., et al. (2010a), Detection of terrestrial gamma ray flashes up to $40 \mathrm{MeV}$ by the AGILE satellite, J. Geophys. Res., 115, A00E13, doi:10.1029/2009JA014502.

Marisaldi, M., et al. (2010b), Gamma-ray localization of terrestrial gamma-ray flashes, Phys. Rev. Lett., 105, 128501, doi:10.1103/ PhysRevLett.105.128501.

Marisaldi, M., et al. (2014), Properties of terrestrial gamma ray flashes detected by AGILE MCAL below 30 MeV, J. Geophys. Res. Space Physics, 119, 1337-1355, doi:10.1002/2013JA019301.

Moore, C. B., K. B. Eack, G. D. Aulich, and W. Rison (2001), Energetic radiation associated with lightning stepped-leaders, Geophys. Res. Lett., 28, 2141-2144, doi:10.1029/2001GL013140.

Moss, G. D., V. P. Pasko, N. Liu, and G. Veronis (2006), Monte Carlo model for analysis of thermal runaway electrons in streamer tips in transient luminous events and streamer zones of lightning leaders, J. Geophys. Res., 111, A02307, doi:10.1029/2005JA011350.

Nisi, R. S., N. Østgaard, T. Gjesteland, and A. B. Collier (2014), An altitude and distance correction to the source fluence distribution of TGFs, J. Geophys. Res. Space Physics, 119, 8698-8704, doi:10.1002/2014JA019817.

Østgaard, N., T. Gjesteland, J. Stadsnes, P. H. Connell, and B. Carlson (2008), Production altitude and time delays of the terrestrial gamma flashes: Revisiting the Burst and Transient Source Experiment spectra, J. Geophys. Res., 113, A02307, doi:10.1029/2007JA012618.

Østgaard, N., T. Gjesteland, R. S. Hansen, A. B. Collier, and B. Carlson (2012), The true fluence distribution of terrestrial gamma flashes at satellite altitude, J. Geophys. Res., 117, A03327, doi:10.1029/2011JA017365.

Østgaard, N., T. Gjesteland, B. E. Carlson, A. B. Collier, S. A. Cummer, G. Lu, and H. J. Christian (2013), Simultaneous observations of optical lightning and terrestrial gamma ray flash from space, Geophys. Res. Lett., 40, 2423-2426, doi:10.1002/grl.50466.

Qin, J., and V. P. Pasko (2014), On the propagation of streamers in electrical discharges, J. Phys. D: Appl. Phys., 435202 , doi:10.1088/0022-3727/47/43/435202.

Rakov, V. A., and M. A. Uman (2003), Lightning: Physics and Effects, Cambridge Univ. Press, Cambridge, U. K., and New York.

Saleh, Z., J. Dwyer, J. Howard, M. Uman, M. Bakhtiari, D. Concha, M. Stapleton, D. Hill, C. Biagi, and H. Rassoul (2009), Properties of the X-ray emission from rocket-triggered lightning as measured by the Thunderstorm Energetic Radiation Array (TERA), J. Geophys. Res., 114, D17210, doi:10.1029/2008JD011618.

Schaal, M. M., J. R. Dwyer, Z. H. Saleh, H. K. Rassoul, J. D. Hill, D. M. Jordan, and M. A. Uman (2012), Spatial and energy distributions of X-ray emissions from leaders in natural and rocket triggered lightning, J. Geophys. Res., 117, D15201, doi:10.1029/2012JD017897.

Schaal, M. M., et al. (2014), The structure of X-ray emissions from triggered lightning leaders measured by a pinhole-type X-ray camera, J. Geophys. Res. Atmos., 119, 982-1002, doi:10.1002/2013JD020266. 
Shao, X.-M., T. Hamlin, and D. M. Smith (2010), A closer examination of terrestrial gamma-ray flash-related lightning processes, J. Geophys. Res., 115, A00E30, doi:10.1029/2009JA014835.

Smith, D. M., L. I. Lopez, R. P. Lin, and C. P. Barrington-Leigh (2005), Terrestrial gamma-ray flashes observed up to $20 \mathrm{MeV}$, Science, 307, $1085-1088$.

Smith, D. M., et al. (2011), A terrestrial gamma ray flash observed from an aircraft, J. Geophys. Res., 116, D20124, doi:10.1029/2011JD016252.

Stanley, M. A., X.-M. Shao, D. M. Smith, L. I. Lopez, M. B. Pongratz, J. D. Harlin, M. Stock, and A. Regan (2006), A link between terrestrial gamma-ray flashes and intracloud lightning discharges, Geophys. Res. Lett., 33, L06803, doi:10.1029/2005GL025537.

Tavani, M., et al. (2011), Terrestrial gamma-ray flashes as powerful particle accelerators, Phys. Rev. Lett., 106, 18501, doi:10.1103/ PhysRevLett.106.018501.

Wilson, C. T. R. (1925), The acceleration of $\beta$-particles in strong electric fields such as those of thunderclouds, Proc. of the Cam. Phil. Soc., 22, 534, doi:10.1017/S0305004100003236.

$\mathrm{Xu}, \mathrm{W}$. S. Celestin, and V. P. Pasko (2012), Source altitudes of terrestrial gamma-ray flashes produced by lightning leaders, Geophys. Res. Lett., 39, L08801, doi:10.1029/2012GL051351.

Xu, W., S. Celestin, and V. P. Pasko (2014), Modeling of X-ray emissions produced by stepping lightning leaders, Geophys. Res. Lett., 41, 7406-7412, doi:10.1002/2014GL061163.

Xu, W., S. Celestin, and V. P. Pasko (2015), Optical emissions associated with terrestrial gamma ray flashes, J. Geophys. Res., 42, 1355-1370, doi:10.1002/2014JA020425. 